\title{
An Oversampling-Based Correlator-Type Receiver for DCSK Communication Systems over Generalized Flat Rayleigh Fading Channels
}

\author{
Nguyen Xuan Quyen \\ Correspondence: quyen.nguyenxuan@hust.edu.vn \\ Communication: received 20 January 2016, revised 19 April 2016, accepted 12 May 2016 \\ Online publication: 16 June 2016, Digital Object Identifier: 10.21553/rev-jec.82 \\ The associate editor coordinating the review of this article and recommending it for publication was Dr. Truong Trung Kien
}

Sch. Electronics \& Telecommunications, Hanoi University of Science and Technology, Hanoi, Vietnam

\begin{abstract}
This paper proposes an oversampling-based correlator-type receiver for Differential Chaos-shift Keying (DCSK) communication systems, which can exploit the flat-fading characteristic of multipath channels in order to improve the system performance. At the receiver, the incoming signal is sampled with a sampling rate higher than chip rate before feeding to a correlator. This oversampling step aims to specifically determine delayed-signal components from fading multipath channels, which can be combined together by the correlator in order to increase the ratio of signal-to-noise at its output. In particular, the performance of the proposed receiver is investigated by means of a generalized flat Rayleigh fading channel which has one primary path (i.e., the path having the shortest transmission period) and multiple secondary paths (i.e., the other remaining paths with delays). Mathematical models in discrete-time domain for the conventional transmitter, generalized channel, and proposed receiver are proposed and analyzed. The theoretical bit-error-rate (BER) expression is first derived and then distribution histogram for the ratio of variable bit energy to noise power spectral density is computed. The BER performance is finally estimated by integrating the BER expression over all possible values of the histogram. Numerical simulations with specific parameters are carried out and then simulated performances are shown in comparison to estimated ones. Obtained results point out that the system performance is significantly improved when the number of secondary paths increases.
\end{abstract}

Keywords- Chaos, Chaos-based communications, differential chaos-shift keying, DCSK, flat fading channel, Rayleigh distribution, BER performance.

\section{INTRODUCTION}

Chaos-based digital communication systems have received strong interest from researchers worldwide over the past decade $[1,2]$. This is mainly due to the observation that chaotic signals not only can be simply generated [3] but also have some beneficial characteristics, i.e., the non-periodic random behavior for increasing the physical-layer security [4-6], the good correlation properties for spread-spectrum and multiple-access performances $[7,8]$, and the immunity of the system to multipath degradation and self-interference $[9,10]$.

Among various digital communication systems using chaos, chaos-based direct-sequence code-division multiple access (chaos-based DS-CDMA) [11-16] and differential chaos shift keying (DCSK) [9], [17-19] have been the most widely studied. In the former chaos-based DSCDMA systems, the chaotic signal is used as a spreading sequence to spread the information-bearing signal and the sequence synchronization is carried out in the receiving side for coherent demodulation [20-23]. On the other hand, the DCSK systems with non-coherent receiver do not require the sequence synchronization, nor channel estimation, but only needs symbol or bit rate. Because of its simple structure, the DSCK system is one of the most promising chaos-based communication schemes for hardware implementation [24, 25]. BER performances of the DCSK systems over additive white Gaussian noise (AWGN) channel and multipath fading channel were investigated in [26-28] and then extended in [29-31] to multipath fading channel with delay spread. However, the channel with delay spread used for all these investigations is a two-path Rayleigh fading channel model [32], where the first path with zero delay is considered as the primary path and the other is the secondary path with non-zero delay.

Beside the conventional system, several extended DCSK systems have been proposed, such as Frequency-modulated DCSK [9], Permutationbased DCSK [33], Reference-modulated DCSK [34], Multi-carrier DCSK [35], DCSK-automatic repeat request/cooperative automatic repeat request (ARQ/CARQ) [36], Improved DCSK [37], and so on, which are to improve the system characteristics, e.g., data security, data rate, spectrum efficiency, bit error rate (BER), under different transmission environments. In particular, the DCSK systems using multiple-antenna diversity techniques, such as singleinput multiple-output (SIMO) [38] and multiple-input multiple-output (MIMO) [39], have been recently 
proposed for the improvement of BER performance in wireless environments.

In this article, we present a performance study for an oversampling-based correlator-type receiver, which is proposed for the communication of DCSK systems over flat fading channels. This study is motivated by the design of secure physical layer for wireless-based applications with low data rate and low power consumption in small transmission areas, such as Wireless Sensor Networks (WSNs) [40] or Low-Rate Wireless Personal Area Networks (LR-WPANs) [41]. For these applications, DCSK using multiple-antenna diversity is impractical due to the cost and size of the sensors. With the scenario of communication in small areas with low rate, the root-mean-square (RMS) delay spread is less than a chip duration, hence the wireless transmission environment can be considered as a flat fading channel [42]. The application of oversampling technique to improve communication features at the receiving side has been studied in [43-45]. In our scenario, oversampling is used to exploit the flat fading characteristic of the channel in order to increase the signal-to-noise (SNR) at the output of the correlator, aiming to improve the bit-error-rate (BER) performance of the system.

In a previous work [46], the idea of using oversampling for improving the performance of the DCSK receiver over flat Rayleigh fading channels has been proposed and investigated by means of a generalized channel, which has one primary path and multiple secondary paths. However, this investigation was carried out by only numerical simulations. In order to develop our work, this idea is fully studied in this article. Mathematical models in the discrete-time domain for the conventional transmitter, the generalized channel, and the proposed receiver are presented and analyzed. The method of performance estimation is based on both theoretical analysis and numerical computation. A theoretical BER expression is determined by means of Gaussian approximation and then a distribution histogram for the ration of variable bit energy to noise power spectral density is computed [47, 48]. The BER performance is finally estimated by integrating the BER expression over all possible values of the histogram.

The main contributions of this paper can be summarized as follows.

- First, we propose an oversampling-based correlator-type receiver for improving the performance of DCSK communication systems over flat fading channels. Receiver operation is described by way of developing and analyzing the mathematical model of the whole system in the discrete-time domain. Performance estimation is carried out by means of both theoretical derivation and numerical computation. The model and the estimation were verified by simulation results.

- Second, we employ a generalized flat Rayleigh fading channel for the performance investigation of the studied DCSK communication system. A discrete-time model with typical parameters of the channel is presented. The impact of the channel on the system performance is determined in terms of the BER value against these parameters. Interestingly, the obtained results showed that as the number of paths in the channel increases, the better the system performs.

The remainder of this paper is organized in the following way. Section 2 presents and analyzes the discretetime model of the DCSK system under study. The BER expression is theoretically derived in Section 3. In Section 4, the histogram of variable bit energy to noise power spectral density ratio is determined and then employed for the BER estimation. The simulation results are shown in comparison with the estimation ones in Section 5. Our conclusion is given in Section 6.

\section{Discrete-time Model Of System}

In this section, a discrete model of the studied DCSK system is developed and analyzed. Figure refFig1(a) shows the block diagram of the conventional transmitter and generalized flat Rayleigh fading channel, whereas the block diagram of the proposed receiver is displayed in Figure refFig1(b).

\subsection{Conventional DCSK Transmitter}

The scheme for the conventional DCSK transmitter has been presented in $[17,18,26-29]$. The chaotic sequence having chip duration $T_{\mathcal{C}}$ at output of the chaotic generator is produced by means of the repeating transformation of a chaotic map. Each transmitted bit duration, denoted by $T_{b}$, is divided into two equal time intervals. The first interval is used to transmit the chaotic reference sequence. The second one is to send the data-bearing sequence. During the second interval, if a bit " +1 " is transmitted, the chaotic reference sequence is repeated, while an inverted copy of the reference sequence is sent if a bit " -1 " is transmitted. Let us denote, $2 \beta=T_{b} / T_{c}$, i.e., number of chips per bit, as a spreading factor of the system. The output signal $e_{k, l}$ of the transmitter in the $l$-th bit duration is given by

$$
e_{k, l}= \begin{cases}x_{k, l}, & k=1, \ldots, \beta, \\ b_{l} x_{k-\beta, l}, & k=\beta+1, \ldots, 2 \beta,\end{cases}
$$

where $b_{l}$ is binary value of the $l$-th bit, equal to either +1 or $-1, x_{k, l}$ is value of the $k$-th chip in the $l$-th bit in the reference sequence, and $x_{k-\beta, l}$ is the delayed version of $x_{k, l}$.

\subsection{Generalized Flat Rayleigh Fading Channel}

The performances of the conventional DCSK system over the AWGN and multipath fading channels with and without delay spread were studied in [26-30], where the channel model of two-path Rayleigh fading was exploited. The generalized multipath Rayleigh fading channels have been recently used for the performance investigation of Multi-carrier DCSK [35] and Chaos-based DS-CDMA [15] systems. Under the assumption that the path delays are much greater than 


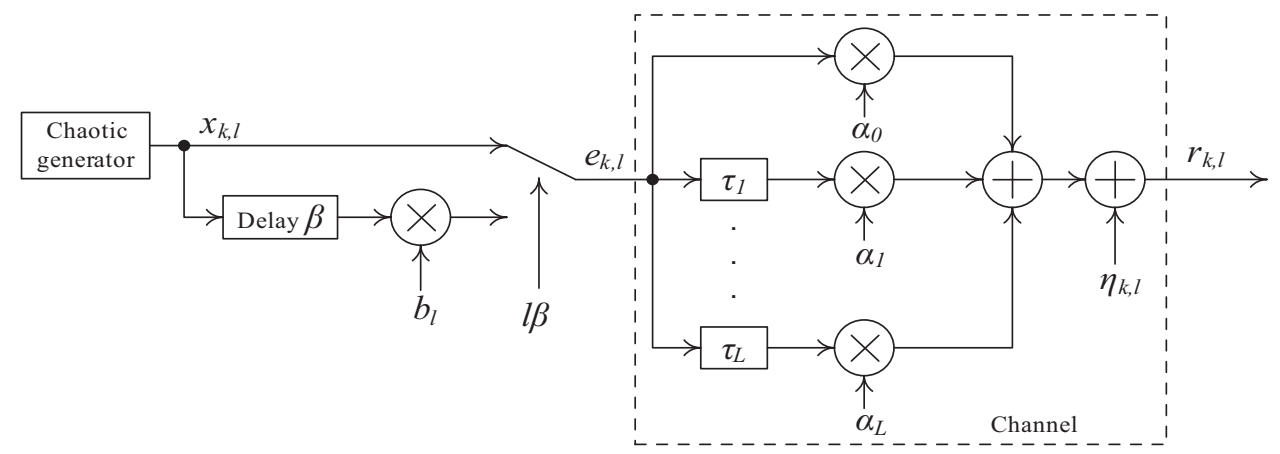

(a)

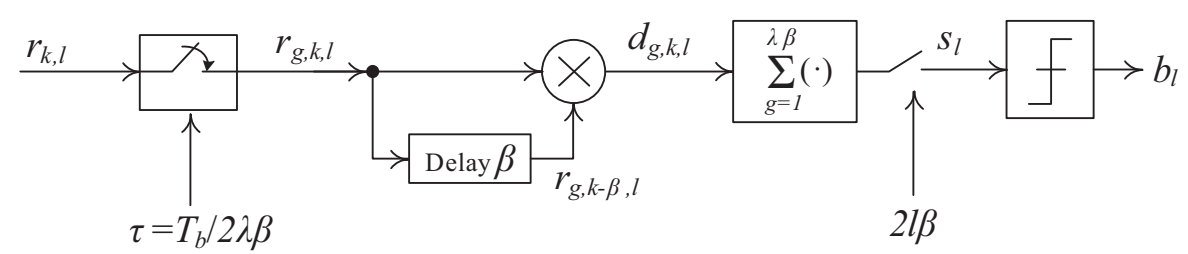

(b)

Figure 1. Block diagram of the DCSK communication system under study: (a) conventional transmitter and the generalized flat Rayleigh fading channel, (b) proposed oversampling-based correlator-type receiver.

the chip duration, these multipath channels have the frequency selective fading characteristic.

In contrast to the above studies, the generalized multipath channel under our investigation is assumed to have the flat (or frequency nonselective) Rayleigh fading characteristic, where all the channel delays are less or equal to the chip duration. In the studied DCSK system, the transmitted signal can reach the receiver from various paths with various delays and attenuation, where the path having a shortest transmission period is considered as the primary path and all others are the secondary paths with non-zero delays. The transmitter and receiver in our system are assumed to be stationary, thus phase variations of the received signals in primary and secondary channels can be ignored.

Let $L$ denote the number of secondary paths, then the impulse response of the channel is given by

$$
h(n)=\sum_{j=0}^{L} \alpha_{j} \delta\left(n-\tau_{j}\right),
$$

where $\delta(t)$ is the Dirac impulse, $\tau_{j}$ and $\alpha_{j}$ are the delay and fading coefficient of the $j$-th path, respectively. The primary path $(j=0)$ has $\alpha_{0}>0$ and $\tau_{0}=0$. The secondary paths $(j=1,2, \ldots, L)$ have $\alpha_{j}>0$ and $\tau_{j}>0$. Here, the fading coefficients $\alpha_{j}$ vary randomly according to the Rayleigh distribution given by

$$
f\left(\alpha_{j}\right)=\frac{\alpha_{j}}{\sigma_{j}^{2}} e^{-\alpha_{j}^{2} /\left(2 \sigma_{j}^{2}\right)},
$$

with $\sigma_{j}$ being the scale parameter of the distribution. The mean value of each fading coefficient is determined by $E\left[\alpha_{j}\right]=\sigma_{j} \sqrt{\pi / 2}$.

Because of the flat fading characteristic, the delays of channel are assumed to satisfy the condition, i.e., $\tau_{1} \leq$ $\tau_{2} \leq \cdots \leq \tau_{L} \leq T_{c}$. Under this condition, the received signal is also the output signal of the channel, which is the sum of the signal components from the primary

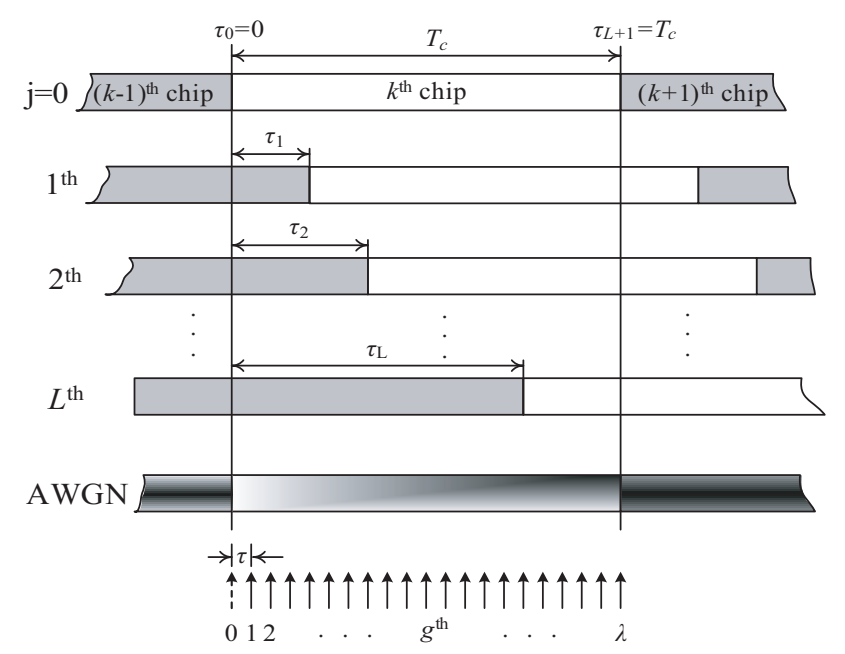

Figure 2. Illustration of the receiving signal components and the sampling process in the receiver.

path with fading and without delay, $L$ secondary paths with both the delay and fading, and AWGN, can be expressed by

$$
r_{k, l}=\alpha_{0} e_{k, l}+\alpha_{1} e_{k-\tau_{1}, l}+\ldots+\alpha_{L} e_{k-\tau_{L}, l}+\eta_{k, l},
$$

where $\alpha_{j} e_{k-\tau_{j}, l}$ is the signal on the $j$-th secondary path and $\eta_{k, l}$ is additive white Gaussian noise (AWGN).

\subsection{Oversampling-based Correlator-type Receiver}

Compared to the conventional DCSK receiver $[17,18$, 26-29], in the proposed receiver an oversampling block is added at its input. This addition causes the remaining blocks (i.e., delay $\beta$, multiplier, sum calculator, sampler, and decision circuit) to work at much higher clock frequency.

Figure 2 illustrates the components of the received signal $r_{k, l}$ and the oversampling process within the duration of $k$-th chip in the second interval of $l$-th 


$$
\begin{aligned}
d_{g, k, l}= & r_{g, k, l} \times r_{g, k-\beta, l} \\
= & \left(\sum_{i=0}^{j} \alpha_{i}\right)^{2} b_{l} x_{k-\beta, l}^{2}+\left(\sum_{i=j+1}^{L} \alpha_{i}\right)^{2} b_{l} x_{(k-1)-\beta, l}^{2}+2 \sum_{i=0}^{j} \alpha_{i} \sum_{i=j+1}^{L} \alpha_{i} b_{l} x_{k-\beta, l} x_{(k-1)-\beta, l}+ \\
& \left(\sum_{i=0}^{j} \alpha_{i} x_{k-\beta, l}+\sum_{i=j+1}^{L} \alpha_{i} x_{(k-1)-\beta, l}\right) \times\left(\eta_{k, l}+b_{l} \eta_{k-\beta, l}\right)+\eta_{k, l} \eta_{k-\beta, l} .
\end{aligned}
$$

$$
\begin{aligned}
s_{l}= & A b_{l} \sum_{k=1}^{\beta} x_{k-\beta, l}^{2}+B b_{l} \sum_{k=1}^{\beta} x_{(k-1)-\beta, l}^{2}+C b_{l} \sum_{k=1}^{\beta} x_{k-\beta, l} x_{(k-1)-\beta, l}+D \sum_{k=1}^{\beta} x_{k-\beta, l}\left(\eta_{k, l}+b_{l} \eta_{k-\beta, l}\right)+ \\
& E \sum_{k=1}^{\beta} x_{(k-1)-\beta, l}\left(\eta_{k, l}+b_{l} \eta_{k-\beta, l}\right)+\lambda \sum_{k=1}^{\beta} \eta_{k, l} \eta_{k-\beta, l},
\end{aligned}
$$

where

$$
\begin{gathered}
A=\sum_{j=0}^{L}\left(\lambda_{j+1}-\lambda_{j}\right)\left(\sum_{i=0}^{j} \alpha_{i}\right)^{2}, B=\sum_{j=0}^{L}\left(\lambda_{j+1}-\lambda_{j}\right)\left(\sum_{i=j+1}^{L} \alpha_{i}\right)^{2}, C=2 \sum_{j=0}^{L}\left(\lambda_{j+1}-\lambda_{j}\right) \sum_{i=0}^{j} \alpha_{i} \sum_{i=j+1}^{L} \alpha_{i}, \\
D=\sum_{j=0}^{L}\left(\lambda_{j+1}-\lambda_{j}\right) \sum_{i=0}^{j} \alpha_{i}, \quad E=\sum_{j=0}^{L}\left(\lambda_{j+1}-\lambda_{j}\right) \sum_{i=j+1}^{L} \alpha_{i} .
\end{gathered}
$$

bit. In the considered duration, we can find that the received signal consists of three components, i.e., the $k$ th chip and its delayed parts with duration of $\left(T_{c}-\tau_{j}\right)$, the delayed parts with duration $\tau_{j}$ of the $(k-1)$-th chip, and AWGN. It also can be found that all samples in the duration of $\left(t_{j}, t_{j+1}\right]$ have the same value. The oversampling block at the input operates at a sampling cycle, i.e., $\tau=T_{b} / 2 \beta \lambda=T_{c} / \lambda$, with $\lambda$ being number of samples in each chip duration $T_{c}$. With no loss of generality, the chip duration $T_{c}$ and all channel delays $\tau_{j}$ are assumed to be equal to a multiple of the sampling cycle $\tau$. For the sake of mathematical representation, we denote, $\lambda_{j}=\tau_{j} / \tau$ and $\tau_{L+1}=T_{c}$. It means that there are $\lambda$ samples in each chip duration $T_{c}$ and $\lambda_{j}$ samples in the duration of $\tau_{j}$. The value of samples falling into the duration of $\left(t_{j}, t_{j+1}\right]$ is determined by

$$
\begin{aligned}
r_{g, k, l} & =\alpha_{0} e_{k, l}+\cdots+\alpha_{j} e_{k, l}+\alpha_{j+1} e_{k-1, l}+\cdots+\alpha_{L} e_{k-1, l}+\eta_{k, l} \\
& =e_{k, l} \sum_{i=0}^{j} \alpha_{i}+e_{k-1, l} \sum_{i=j+1}^{L} \alpha_{i}+\eta_{k, l} \\
& =b_{l} x_{k-\beta, l} \sum_{i=0}^{j} \alpha_{i}+b_{l} x_{(k-1)-\beta, l} \sum_{i=j+1}^{L} \alpha_{i}+\eta_{k, l}
\end{aligned}
$$

where $r_{g, k, l}$ is the value of $g$-th sample in the $k$-th chip duration, satisfying $\lambda_{j}<g \leq \lambda_{j+1}$.

Similarly, we can express the value of samples in $\left(t_{j}, t_{j+1}\right]$ of the signal at the output of the delay $\beta$ block as follows:

$$
r_{g, k-\beta, l}=x_{k-\beta, l} \sum_{i=0}^{j} \alpha_{i}+x_{(k-1)-\beta, l} \sum_{i=j+1}^{L} \alpha_{i}+\eta_{k-\beta, l} .
$$

Based on the results in (8) and (9), the value of samples in $\left(t_{j}, t_{j+1}\right]$ at the output of the multiplier is determined by

$$
d_{g, k, l}=r_{g, k, l} \times r_{g, k-\beta, l},
$$

which is further expressed in Equation (5) in the next page.

In the sum block, the consecutive samples at input are added together in the second half of each bit duration to produce a decision variable $s_{l}$ as follows:

$$
\begin{aligned}
s_{l} & =\sum_{g=1}^{\beta \lambda} d_{g, k, l} \\
& =\sum_{k=1}^{\beta} \sum_{g=1}^{\lambda} d_{g, k, l} \\
& =\sum_{k=1}^{\beta} \sum_{j=0}^{L}\left(\lambda_{j+1}-\lambda_{j}\right) d_{g, k, l},
\end{aligned}
$$

which yields (6) at the top of this page.

We can find that the signal $s_{l}$ contains the following components. The component of the beneficial signal denoted by

$$
W=A b_{l} \sum_{k=1}^{\beta} x_{k-\beta, l}^{2}+B b_{l} \sum_{k=1}^{\beta} x_{(k-1)-\beta, l}^{2}
$$

The interference component between the current chip with its delayed version, denoted by

$$
X=C b_{l} \sum_{k=1}^{\beta} x_{k-\beta, l} x_{(k-1)-\beta, l} .
$$

The noise component created by the impact of the AWGN, denoted by

$$
\begin{aligned}
Y= & D \sum_{k=1}^{\beta} x_{k-\beta, l}\left(\eta_{k, l}+b_{l} \eta_{k-\beta, l}\right)+ \\
& E \sum_{k=1}^{\beta} x_{(k-1)-\beta, l}\left(\eta_{k, l}+b_{l} \eta_{k-\beta, l}\right) .
\end{aligned}
$$


The noise component created by the AWGN only, denoted by

$$
Z=\lambda \sum_{k=1}^{\beta} \eta_{k, l} \eta_{k-\beta, l}
$$

With respect to the components in the output signal $s_{l}$ expressed by Equation (6), $W$ is the beneficial component, while the sum $(X+Y+Z)$ is the negative component created by interference and noise. It can be seen from Equation (7) that the values of $A, B$, $C, D$ increase when the number of delay paths $L$ or the number of samples per chip $\lambda$ increases. On the other hand, Equations (12), (13), (14), and (15) show that the value increment of $A, B, C, D$ leads to the value increment of the components $W, X, Y$, and $Z$. However, owing to the low cross-correlation between the chaotic sequences with different delays, between the chaotic sequences and AWGN, and between AWGN and its delay, as well as the high auto-correlation between the same chaotic sequence, the increasing amount of the components $X, Y$ and $Z$ is much less than that of the component $W$. Therefore, the ratio of the beneficial and negative components, which can be considered as the SNR of $s_{l}$, is increased.

Finally, the binary value of the $l$-th bit is recovered according to the following rule:

$$
b_{l}= \begin{cases}1, & s_{l} \geq 0, \\ -1, & s_{l}<0 .\end{cases}
$$

\section{Theoretical BER Expression}

This section presents the theoretical derivation of the system performance with the use of statistics and Gaussian approximation. First, the statistics of the components, i.e., $W, X, Y$ and $Z$, in case of a " +1 " bit transmitted are determined. Under the assumption that the value of $\beta$ is high enough so that the correlation values of the independent elements in these components are approximately equal to zero. The mean and second moment values are determined as follows (see Appendix A):

$$
\begin{aligned}
E\left[W \mid b_{l}=+1\right] & =(A+B) \beta E_{c}, \\
E\left[X \mid b_{l}=+1\right]= & E\left[Y \mid b_{l}=+1\right] \\
= & E\left[Z \mid b_{l}=+1\right]=0, \\
E\left[W^{2} \mid b_{l}=+1\right]= & \left(A^{2}+B^{2}\right) \beta\left(E_{c 4}+(\beta-1) E_{c}^{2}\right)+ \\
& 2 A B \beta^{2} E_{c}^{2} \\
E\left[X^{2} \mid b_{l}=+1\right]= & C^{2} \beta E_{c}^{2} \\
E\left[Y^{2} \mid b_{l}=+1\right]= & \left(D^{2}+E^{2}\right) \beta N_{0} E_{c}, \\
E\left[Z^{2} \mid b_{l}=+1\right]= & \lambda^{2} \beta \frac{N_{0}^{2}}{4}
\end{aligned}
$$

where $E_{c}=E\left[x_{k, l}^{2}\right]$ and $E_{c 4}=E\left[x_{k, l}^{4}\right]$.

Based on the results above, the variances of $W, X, Y$ and $Z$ are calculated by

$$
\begin{aligned}
\operatorname{Var}\left[W \mid b_{l}=+1\right] & =E\left[W^{2} \mid b_{l}=+1\right]-E^{2}\left[W \mid b_{l}=+1\right] \\
& =\left(A^{2}+B^{2}\right) \beta\left(E_{c 4}-E_{c}^{2}\right) \\
\operatorname{Var}\left[X \mid b_{l}=+1\right] & =E\left[X^{2} \mid b_{l}=+1\right]-E^{2}\left[X \mid b_{l}=+1\right] \\
& =C^{2} \beta E_{c}^{2} \\
\operatorname{Var}\left[Y \mid b_{l}=+1\right] & =E\left[Y^{2} \mid b_{l}=+1\right]-E^{2}\left[Y \mid b_{l}=+1\right] \\
& =\left(D^{2}+E^{2}\right) \beta N_{0} E_{c} \\
\operatorname{Var}\left[Z \mid b_{l}=+1\right] & =E\left[Z^{2} \mid b_{l}=+1\right]-E^{2}\left[Z \mid b_{l}=+1\right] \\
& =\lambda^{2} \beta \frac{N_{0}^{2}}{4}
\end{aligned}
$$

Due to the statistical independence between Rayleigh fading coefficients, secondary path delays, Gaussian noise and chaotic sequences, the components (i.e., $W, X$, $Y, Z)$ are also considered as the independent variables. Therefore, the mean value and variance of the decision variable $s_{l}$ are respectively determined as

$$
\begin{aligned}
& E\left[s_{l} \mid b_{l}=+1\right]= E\left[W \mid b_{l}=+1\right]+E\left[X \mid b_{l}=+1\right]+ \\
& E\left[Y \mid b_{l}=+1\right]+E\left[Z \mid b_{l}=+1\right] \\
&= E\left[W \mid b_{l}=+1\right], \\
& \operatorname{Var}\left[s_{l} \mid b_{l}=+1\right]= \operatorname{Var}\left[W \mid b_{l}=+1\right]+\operatorname{Var}\left[X \mid b_{l}=+1\right]+ \\
& \operatorname{Var}\left[Y \mid b_{l}=+1\right]+\operatorname{Var}\left[Z \mid b_{l}=+1\right] .
\end{aligned}
$$

Similarly, for the case of a " -1 " bit being transmitted, we have

$$
\begin{aligned}
E\left[s_{l} \mid b_{l}\right. & =-1]=-E\left[s_{l} \mid b_{l}=+1\right], \\
\operatorname{Var}\left[s_{l} \mid b_{l}\right. & =-1]=\operatorname{Var}\left[s_{l} \mid b_{l}=+1\right] .
\end{aligned}
$$

Assuming that each bit, either " +1 " or " -1 ", appears at the output of the data source with a probability of $1 / 2$. Based on the obtained results above, the BER expression can be derived by means of the Gaussian approximation as given by (31), where the function $Q(\cdot)$ is defined by

$$
Q(\epsilon)=\frac{1}{\sqrt{2 \pi}} \int_{\epsilon}^{\infty} \exp \left(y^{2} / 2\right) d y,
$$

$E_{b}=\beta E_{c}$ is known as energy per bit, and $N_{0}=2 E\left[\eta_{k, l}^{2}\right]$ is noise power spectral density.

In our DCSK system, since the the fading coefficients vary randomly according to the Rayleigh distribution, three variables in (31), denoted by,

$$
\begin{aligned}
F & =\frac{\left(A^{2}+B^{2}\right)\left(\frac{E_{c 4}}{E_{c}^{2}}-1\right)+C^{2}}{\beta(A+B)^{2}}, \\
G & =\frac{2\left(D^{2}+E^{2}\right)}{(A+B) \lambda}, \\
H & =\frac{A+B}{\lambda} \frac{E_{b}}{N_{0}},
\end{aligned}
$$

also randomly vary in the communication process.

Here, the variable $H$ is considered as the ratio of variable bit energy, i.e.,

$$
E_{v b}=\frac{(A+B) E_{b}}{\lambda}
$$




$$
\begin{aligned}
\mathrm{BER} & =\frac{1}{2} \operatorname{Pr}\left(s_{l} \leq 0 \mid b_{l}=+1\right)+\frac{1}{2} \operatorname{Pr}\left(s_{l}>0 \mid b_{l}=-1\right) \\
& =\operatorname{Pr}\left(s_{l} \leq 0 \mid b_{l}=+1\right)=Q\left(\frac{\operatorname{Var}\left[s_{l} \mid b_{l}=+1\right]}{E^{2}\left[s_{l} \mid b_{l}=+1\right]}\right)^{-\frac{1}{2}} \\
& =Q\left(\frac{\left(A^{2}+B^{2}\right)\left(\frac{E_{c 4}}{E_{c}^{2}}-1\right)+C^{2}}{\beta(A+B)^{2}}+\frac{2\left(D^{2}+E^{2}\right)}{(A+B)^{2} \frac{E_{b}}{N_{0}}}+\frac{\lambda^{2} \beta}{(A+B)^{2}\left(\frac{E_{b}}{N_{0}}\right)^{2}}\right)^{-\frac{1}{2}}
\end{aligned}
$$

to noise power spectral density $N_{0}$. We can find that $E_{v b}$ depends on the constant bit energy $E_{b}$ and the parameters of the channel, i.e., $L, \alpha_{j}, \tau_{j}$. In order to simplify our estimation, the variables $F$ and $G$ are considered as constants which are respectively equal to

$$
\begin{aligned}
& \bar{F}=\frac{\left(\bar{A}^{2}+\bar{B}^{2}\right)\left(\frac{E_{c 4}}{E_{c}^{2}}-1\right)+\bar{C}^{2}}{\beta(\bar{A}+\bar{B})^{2}}, \\
& \bar{G}=\frac{\left(\bar{D}^{2}+\bar{E}^{2}\right)}{(\bar{A}+\bar{B}) \lambda},
\end{aligned}
$$

where the constants $\bar{A}, \bar{B}, \bar{C}, \bar{D}, \bar{E}$ are respectively obtained by replacing the variable coefficients $\alpha_{j}$ in $A$, $B, C, D, E$ by their mean values, i.e., $\bar{\alpha}_{j}=\sigma_{j} \sqrt{\pi / 2}$.

The BER expression is first approximated by

$$
\mathrm{BER} \approx Q\left(\bar{F}+\frac{\bar{G}}{H}+\frac{\beta}{H^{2}}\right)^{-\frac{1}{2}} .
$$

Then, the conditional BER expression reflecting the variation of fading coefficients is calculated as follows:

$$
\operatorname{BER}(H) \approx \int_{0}^{\infty} Q\left(\bar{F}+\frac{\bar{G}}{H}+\frac{\beta}{H^{2}}\right)^{-\frac{1}{2}} f(H) d H
$$

with $f(H)$ being the probability density function (PDF) of $H$.

It is noted that the BER expression in (33) is derived from that in (31) which is a function of three variables, i.e., $F, G$, and $H$. If we divide both the numerator and denominator of these variables by the same constant $\lambda$, they will be as follows:

$$
\begin{aligned}
F & =\frac{\left(\left(\frac{A}{\lambda}\right)^{2}+\left(\frac{B}{\lambda}\right)^{2}\right)\left(\frac{E_{c 4}}{E_{c}^{2}}-1\right)+\left(\frac{C}{\lambda}\right)^{2}}{\beta\left(\frac{A}{\lambda}+\frac{B}{\lambda}\right)^{2}}, \\
G & =2 \frac{\left(\frac{D}{\lambda}\right)^{2}+\left(\frac{E}{\lambda}\right)^{2}}{\left(\frac{A}{\lambda}+\frac{B}{\lambda}\right)}, \\
H & =\left(\frac{A}{\lambda}+\frac{B}{\lambda}\right) \frac{E_{b}}{N_{0}} .
\end{aligned}
$$

Based on the expressions of $A, B, C, D$ in (7), it is easy to find that the fractions, i.e., $A / \lambda, B / \lambda, C / \lambda$, $D / \lambda$, depend on the ratio of $\lambda_{j} / \lambda$. Therefore, the BER expression in (33) just depends on the value of this ratio, but not on the specific values of $\lambda_{j}$ or $\lambda$.

Let us now consider the above conditional BER expression in some special cases of the channel.
AWGN channel: It is equivalent to our channel in the case of $L=0$ and $\alpha_{0}=1$. We have $\bar{F}=0, \bar{G}=2$ and $H=E_{b} / N_{0}$. The BER expression is shorten as

$$
\operatorname{BER}\left(\frac{E_{b}}{N_{0}}\right) \approx Q\left(\frac{2}{\frac{E_{b}}{N_{0}}}+\frac{\beta}{\left(\frac{E_{b}}{N_{0}}\right)^{2}}\right)^{-\frac{1}{2}} .
$$

This BER result is the same as the obtained expression in [27].

One-path Rayleigh fading channel: This channel has been used for performance investigation of the conventional DCSK system in [34] and it is equivalent to our channel in the case of $L=0$ and $\alpha_{0}>0$. Under this condition, we have $\bar{F}=0, \bar{G}=2, H=\alpha_{0}^{2} \frac{E_{b}}{N_{0}}$ and $f(H)=\frac{1}{\bar{H}} e^{-H / \bar{H}}$ with $\bar{H}=E\left[\alpha_{0}^{2}\right] \frac{E_{b}}{N_{0}}=2 \sigma_{0}^{2} \frac{E_{b}}{N_{0}}$. The BER expression becomes

$$
\operatorname{BER}(H) \approx \int_{0}^{\infty} Q\left(\frac{2}{H}+\frac{\beta}{H^{2}}\right)^{-\frac{1}{2}} \frac{1}{\bar{H}} e^{-H / \bar{H}} d H .
$$

This result is also the BER expression obtained in [28].

The special cases above point out that the conditional BER expression obtained totally agrees with the results in the previous studies. However, the more the number of secondary paths $L$ increases, the more complicated, if not impossible, the theoretical determination of the PDF $f(H)$ is. For this reason, the use of numerical computation to determine the histogram of the value distribution of $H$ instead of theoretically determining its PDF is presented in the next section.

\section{Distribution Histogram for BER ESTIMATION}

In this section, the histogram of the value distribution of the component $H$ is built by means of the numerical computation. Firstly, the total number of samples $M$ for the statistic computation and the number of classes $N$ for building the histogram are chosen. Due to the fading coefficient of each path having a given scale parameter $\sigma_{j}$, by using a numerically programmable tool (e.g., MATLAB), we generate $M$ discrete samples for each corresponding coefficient, which are expressed by a $M$-element vector,

$$
\vec{\alpha}^{(j)}=\left[\begin{array}{llll}
\alpha_{1}^{(j)} & \alpha_{2}^{(j)} & \ldots & \alpha_{M}^{(j)}
\end{array}\right]
$$




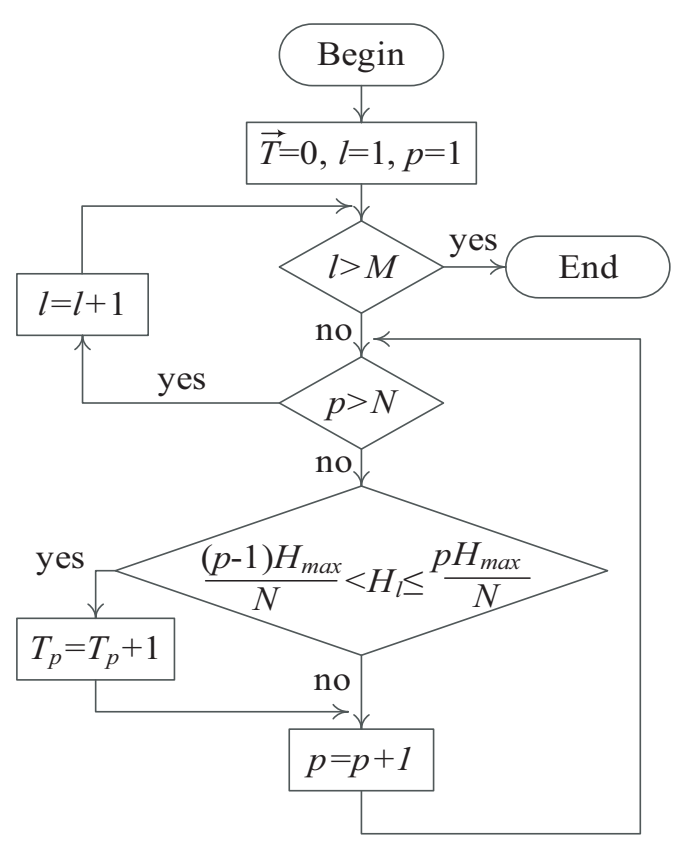

Figure 3. Algorithm for the computation of $\mathrm{N}$ elements in the vector $\vec{T}$ from the total number of $M$ samples generated.

and then based on Equation (7), the vectors $\vec{A}$ and $\vec{B}$, are created by

$$
\begin{gathered}
\vec{A}=\left[\sum_{j=0}^{L}\left(\lambda_{j+1}-\lambda_{j}\right)\left(\sum_{i=0}^{j} \alpha_{1}^{(i)}\right)^{2}, \ldots,\right. \\
\left.\sum_{j=0}^{L}\left(\lambda_{j+1}-\lambda_{j}\right)\left(\sum_{i=0}^{j} \alpha_{M}^{(i)}\right)^{2}\right], \\
\vec{B}=\left[\sum_{j=0}^{L}\left(\lambda_{j+1}-\lambda_{j}\right)\left(\sum_{i=j+1}^{L} \alpha_{1}^{(i)}\right)^{2}, \ldots,\right. \\
\left.\sum_{j=0}^{L}\left(\lambda_{j+1}-\lambda_{j}\right)\left(\sum_{i=j+1}^{L} \alpha_{M}^{(i)}\right)^{2}\right] .
\end{gathered}
$$

Using the obtained vectors, i.e., $\vec{A}$ and $\vec{B}$, the vector for $M$ discrete samples of the variable $H$ are produced by

$$
\vec{H}=\frac{(\vec{A}+\vec{B})}{\lambda} \frac{E_{b}}{N_{0}} .
$$

In theory, the variation range of the variable $H$ is $(0, \infty)$. However, in our numerical approach, the value variation of elements in the vector $\vec{H}$ is assumed in a limited range of $\left(0, H_{\max }\right]$, where $H_{\max }$ is chosen with a large enough value to guarantee the accurateness of the estimation. The range of $\left(0, H_{\max }\right]$ is divided in to $N$ equal intervals, i.e., $\left(0, H_{\max } / N\right],\left(H_{\max } / N, 2 H_{\max } / N\right], \ldots,\left(p H_{\max } / N\right)(p+$ 1) $\left.H_{\max } / N\right], \ldots,\left((N-1) H_{\max } / N, N H_{\max } / N\right]$. The number of samples, denoted by element $T_{p}$ in vector $\vec{T}$, from $M$ samples in the vector $\vec{H}$ having value falling into each corresponding interval $((p-$ 1) $\left.H_{\max } / N, p H_{\max } / N\right]$ are calculated by the algorithm as in Figure 3. Based on the vector $\vec{T}$ obtained, the element $P_{p}$ of the vector $\vec{P}$, which denotes the probability that the variable $H$ has value in the interval,

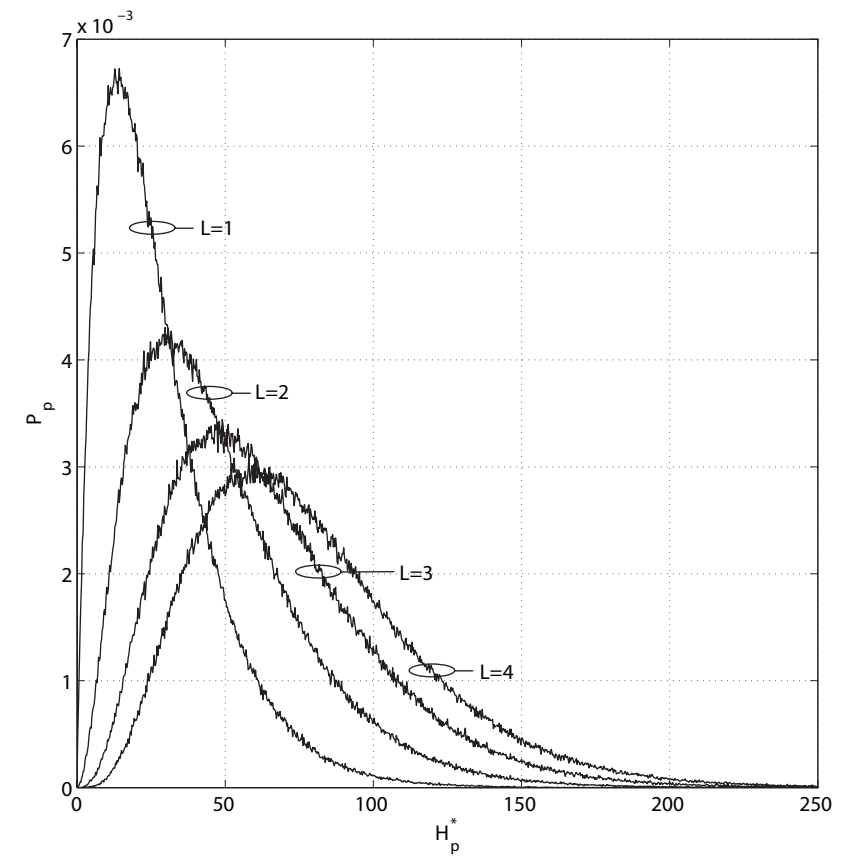

Figure 4. Histograms of value distribution of the variable $H$.

$\left((p-1) H_{\max } / N, p H_{\max } / N\right]$, is computed by

$$
\vec{P}=\frac{\vec{T}}{M} .
$$

Notice that, $\vec{T}$ and $\vec{P}$ are $N$-element vectors and $\sum_{p=1}^{N} P_{p}=1$.

The histogram of value distribution of variable $H$ is created by plotting $P_{p}$ versus the centred value of each interval, i.e., $H_{p}^{*}=(p-1 / 2) H_{\max } / N$ for $p$ from 1 to $N$. The BER estimation is finally carried out by discretely integrating the expression in Equation (32) over all $N$ classes of the histogram as follows:

$$
\mathrm{BER} \approx \sum_{p=1}^{N} Q\left(\bar{F}+\frac{\bar{G}}{H_{p}^{*}}+\frac{\beta}{\left(H_{p}^{*}\right)^{2}}\right)^{-\frac{1}{2}} P_{p} .
$$

Figure 4 shows the histograms obtained by the above computation with the number of secondary paths $L$ changing from 1 to 4 . Here, the delays are fixed while the fading coefficients randomly vary according to the Rayleigh distribution with different scale parameters. The channel parameters are set as follows: $\sigma_{0}=0.7$ for the primary path, $\sigma_{1}=0.6, \lambda_{1}=5, \sigma_{2}=0.5$, $\lambda_{2}=10, \sigma_{3}=0.4, \lambda_{3}=15, \sigma_{4}=0.3, \lambda_{4}=20$ for the secondary paths, and $E_{b} / N_{0}=10 \mathrm{~dB}$ for the AWGN. The parameters for computation are given as follows: the total number of samples $M=10^{6}$, the number of classes $N=10^{3}$, the maximum value $H_{\max }=250$ and the number of samples in each chip duration $\lambda=30$. It can be seen that when $L$ increases, the value distribution changes. Specifically, the average value of $H_{p}^{*}$ also increases while the average probability of $P_{p}$ reduces. Based on Equation (41), we can find that this change will make the BER reduce.

It is noticed that the higher the values of $M$ and $N$ are chosen, the more accurate the BER estimation is, however the more the computation time takes and vice 


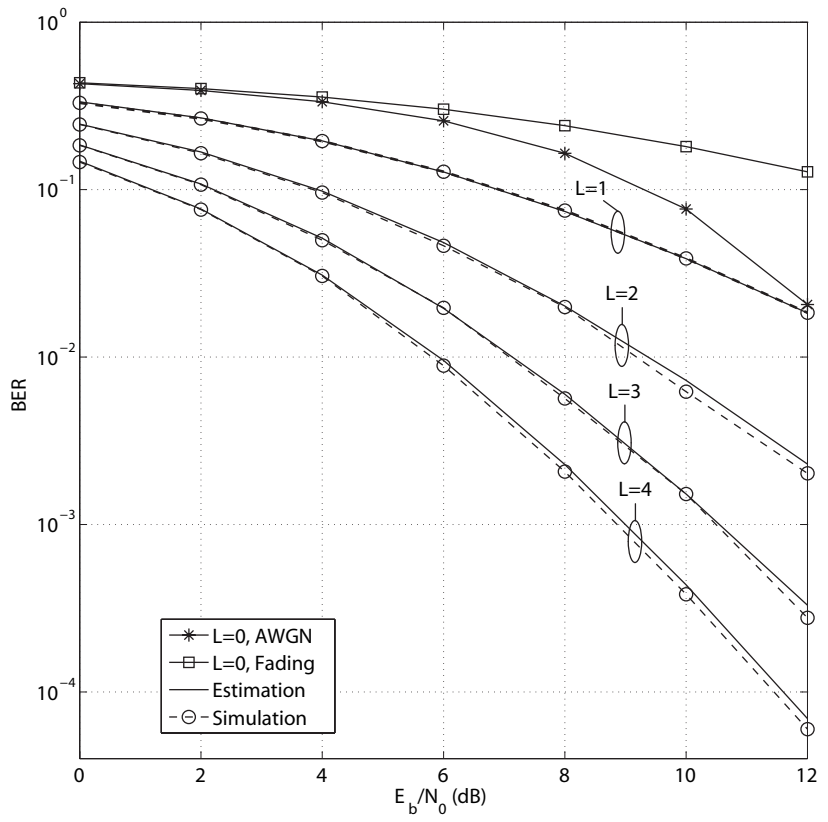

Figure 5. BER values versus the ratio $E_{b} / N_{0}$.

versa. Therefore, the value choice of $M$ and $N$ has to guarantee an acceptable accurateness with an allowable period of computation time.

\section{Simulation Results}

In this section, numerical results obtained by simulations with different specific parameters are presented in comparison with the estimation results. The dependence of BER values on the variation of different parameters is shown with the gradual increment of the number of secondary paths. The initial parameters for the generalized channel as well as for building the histogram are set as in Section IV. The spreading factor is chosen with $2 \beta=64$. The chaotic map used for generating the chaotic sequences is the Chebyshev polynomial function of order 2, i.e.,

$$
x_{k}=f\left(x_{k-1}\right)=2 x_{k-1}^{2}-1,
$$

with $E_{c}=1 / 2$ and $E_{c 4}=3 / 8$ (see Appendix B).

\subsection{BER Versus Parameters Of Channel}

BER performances obtained from Equations (34) and (35) for the special cases of channel as well as from the estimation expression in Equation (41) for $L=1,2,3,4$ are presented in Figure 5. The corresponding simulation results are also plotted. It can find that there is a good match between the estimated and simulated results for any value of the ratio $E_{b} / N_{0}$ and the number of secondary paths $L$. We can observe that the system performance is significantly improved when the number of secondary paths increases. For example, at the same value of $E_{b} / N_{0}=10 \mathrm{~dB}$, the BER values corresponding to $L=0,1,2,3,4$ are $1.2 \cdot 10^{-1}, 3.9 \cdot 10^{-2}$, $6.2 \cdot 10^{-3}, 1.5 \cdot 10^{-3}$ and $3.4 \cdot 10^{-4}$, respectively.

In Figure 6, we study the effect of the time delay of each secondary path on the system performance. The

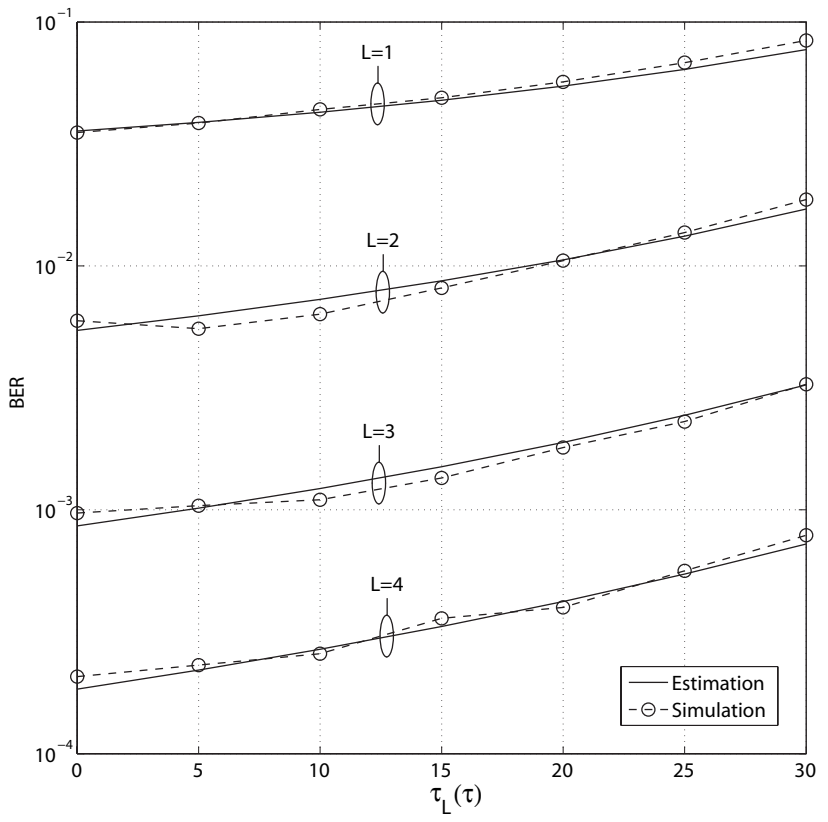

Figure 6. BER values versus the delay $\tau_{L}$ of $L$-th path.

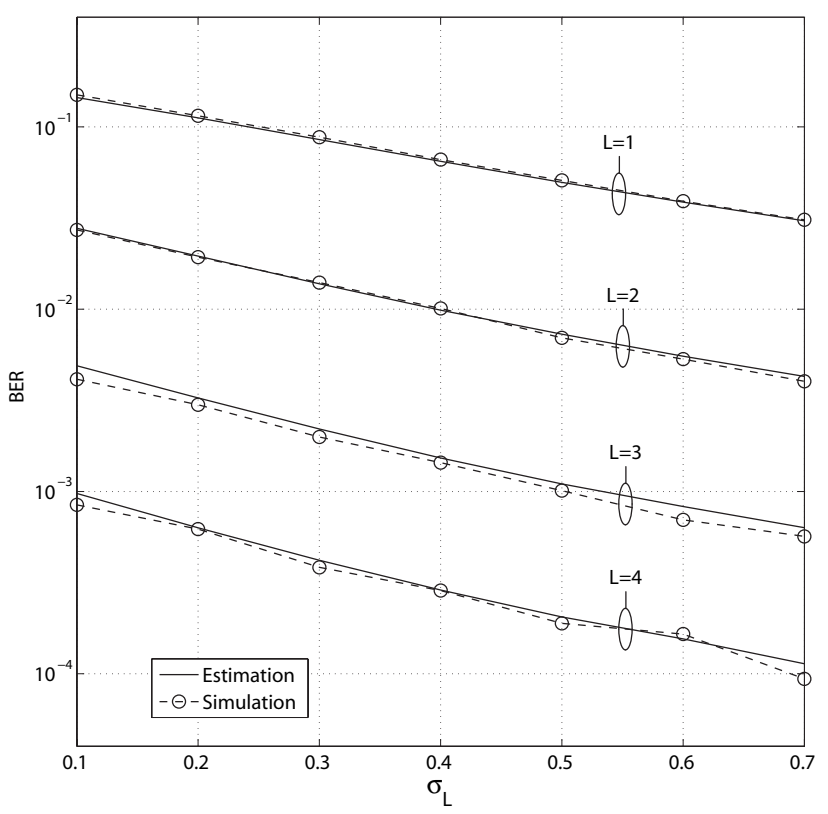

Figure 7. BER values versus the scale parameter $\sigma_{L}$ of $L$-th path.

BER curves are plotted by changing the delay of the $L$-th path, i.e., $\tau_{L}$, from 0 to $T_{c}=\lambda \tau$ while keeping the delays of other paths, i.e., $\tau_{1}, \ldots, \tau_{L-1}$, and the ratio $E_{b} / N_{0}$ unchanged from the initial setting. For example with the case of $L=3$, the delay $\tau_{3}$ is varied from 0 to $30 \tau$ while $\tau_{1}=\lambda_{1} \tau=5 \tau, \tau_{2}=\lambda_{2} \tau=10 \tau$ and $E_{b} / N_{0}=10 \mathrm{~dB}$.

Figure 7 evaluates the effect of the scale parameter of each secondary path on the BER values. In the similar way, the scale parameters, i.e., $\sigma_{0}, \sigma_{1}, \ldots, \sigma_{L-1}$ are kept as the initial setting while the value of $\sigma_{L}$ is changed from 0.1 to 0.7 . It clearly appears that the DCSK system performs better when the delay reduces or the scale parameter increases. 


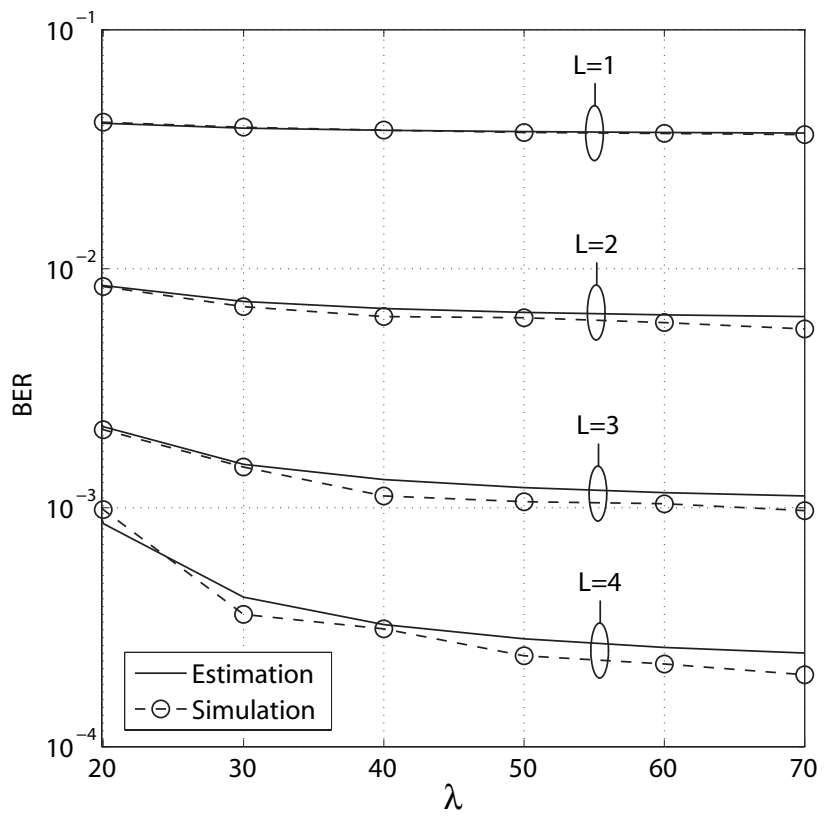

Figure 8. BER values versus the number of samples per chip $\lambda$ in case of keeping the sampling clock fixed and changing the chip duration $T_{\mathcal{c}}$.

\subsection{BER Versus Parameters Of Transmitter And Receiver}

In our DCSK system, with the fixed channel delays $\tau_{j}$, the number of samples per chip $\lambda$ can be changed in two following cases: keeping the chip duration fixed and changing the sampling clock in the receiver, or keeping the sampling clock constant and changing the chip duration.

In the first case, even though the sampling clock changes, the ratio (i.e., $\lambda_{j} / \lambda=\tau_{j} / T_{c}$ ) is still unvaried. Notice in Equation (33) that the BER just depends on the ratio $\lambda_{j} / \lambda$, but not on the specific values of $\lambda_{j}$ or $\lambda$. Therefore, this case does not make the BER changed. In the second case, the simultaneous change of chip duration $T_{\mathcal{C}}$ in both transmitter and receiver leads to the change of ratio $\lambda_{j} / \lambda$ and thus the BER of system also varies. Figure 8 shows the dependence of BER upon $\lambda$ for the second case. The simulation results point out that the system outperforms when $\lambda$ is increased. For example with the case of $L=4$, the BER value reduces from $9.6 \cdot 10^{-4}$ to $3.4 \cdot 10^{-4}$ corresponding to $\lambda$ increases from 20 to 70 . It can be seen that the estimated results totally agree with the simulated ones.

Figure 9 evaluates the effect of the value of the spreading factor on the BER performance. It clearly appears that in the value range from 20 to 98 , the increment of $\beta$ also makes the BER values increase. For example with the case of $L=2$ at the same $E_{b} / N_{0}=10 \mathrm{~dB}$, the simulated BER increases from $7.3 \cdot 10^{-3}$ to $2.1 \cdot 10^{-2}$ with $\beta$ changing from 20 to 98 , respectively. In particular, the BERs achieve their minimum values with the spreading factor between 10 and 20. These results prove that good performances are obtained for low spreading factor values which makes this system implementation feasible even for a moderate bandwidth. Generally, the simulation results

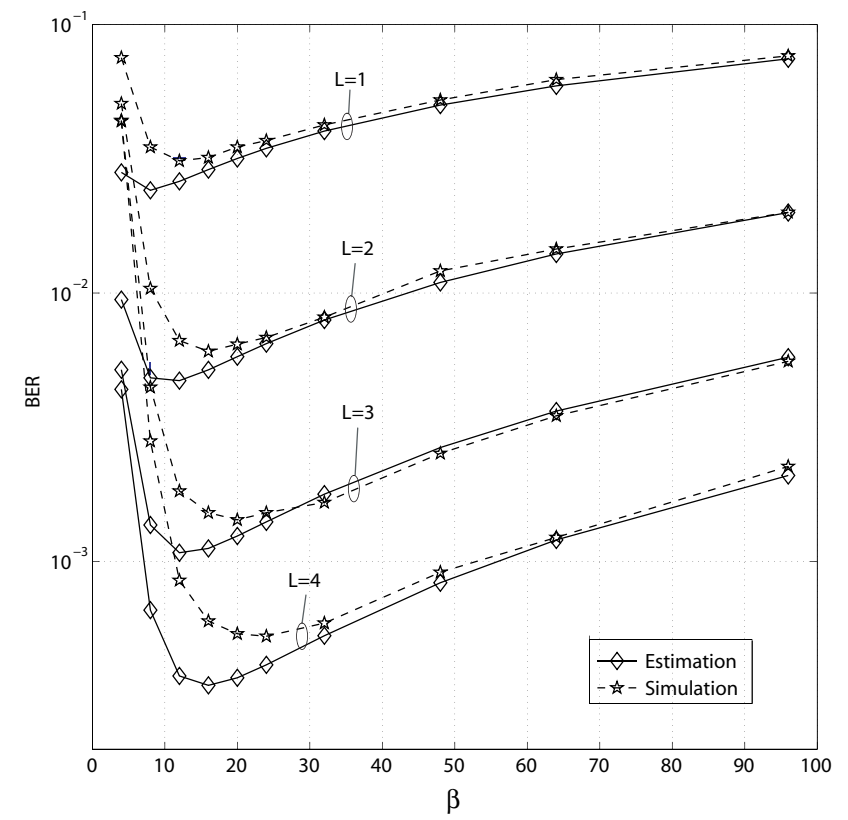

Figure 9. BER values versus the spreading factor $2 \beta$.

agree with the estimation ones. However, at the low values of $\beta$, i.e., less than 20 , slight differences between the estimation and simulation curves start to be visible. These differences become more pronounced at higher values of $L$. The main reasons are that the variables $F$ and $G$ in Equation (31) is approximated respectively by the fixed values $\bar{F}$ and $\bar{G}$ in (32). Notice that these approximations are to make our BER estimation simpler but simultaneously it creates the differences.

\section{Conclusions}

An oversampling-based correlator-type DCSK receiver has been proposed and investigated in this paper. The demodulation operation of the receiver along with the modulation operation of the conventional transmitter and the transmission process over the generalized flat Rayleigh fading channel are described in detail by means of their discrete-time mathematical models. Based on the theoretical BER expression and distribution histogram obtained, the BER performance is estimated by means of the numerical integral. The simulation results agree with the estimation ones. It can be seen from the obtained results that the proposed receiver can perform well in the flat fading channels, especially the BER performance is significantly enhanced when the number of secondary paths increases. In addition, since the receiver can perform the demodulation process based on the discrete-sample processing, it is suitable to be implemented on the programmable integrated circuits (IC) such as FPGA or DSP. Moreover, with the simple structure, the receiver is a promising solution for the design of secure physical layer in the WSNs or LR-WPANs. Last but not least, it is noted that the use of the oversampling block at the input requires that all remaining blocks in the receiver also have to work at much higher clock frequency. This leads 


$$
\begin{aligned}
& E\left[W \mid b_{l}=+1\right]=A \sum_{k=1}^{\beta} E\left[x_{k-\beta, l}^{2}\right]+B \sum_{k=1}^{\beta} E\left[x_{(k-1)-\beta), l}^{2}\right]=(A+B) \beta E_{c}, \\
& E\left[X \mid b_{l}=+1\right]=C \sum_{k=1}^{\beta} E\left[x_{k-\beta, l} x_{(k-1)-\beta), l}\right]=0, \\
& E\left[Y \mid b_{l}=+1\right]=D \sum_{k=1}^{\beta} E\left[x_{k-\beta, l} \eta_{k, l}\right]+D \sum_{k=1}^{\beta} E\left[x_{k-\beta, l} \eta_{k-\beta, l}\right]+E \sum_{k=1}^{\beta} E\left[x_{(k-1)-\beta, l} \eta_{k, l}\right]+E \sum_{k=1}^{\beta} E\left[x_{(k-1)-\beta, l} \eta_{k-\beta, l}\right]=0, \\
& E\left[Z \mid b_{l}=+1\right]=\lambda \sum_{k=1}^{\beta} E\left[\eta_{k, l} \eta_{k-\beta, l}\right]=0 \\
& E\left[W^{2} \mid b_{l}^{(i)}=+1\right]=A^{2} \sum_{k=1}^{\beta} E\left[x_{k-\beta, l}^{4}\right]+A^{2} \sum_{k=1}^{\beta} E\left[x_{k-\beta, l}^{2}\right] \sum_{m=1}^{\beta-1} E\left[x_{m-\beta, l}^{2}\right]+B^{2} \sum_{k=1}^{\beta} E\left[x_{(k-1)-\beta), l}^{2}\right]+ \\
& B^{2} \sum_{k=1}^{\beta} E\left[x_{(k-1)-\beta, l}^{2}\right] \sum_{m=1}^{\beta-1} E\left[x_{(m-1)-\beta, l}^{2}\right]+2 A B \sum_{k=1}^{\beta} E\left[x_{k-\beta, l}^{2}\right] \sum_{k=1}^{\beta} E\left[x_{(k-1)-\beta, l}^{2}\right] \\
& =\left(A^{2}+B^{2}\right) \beta E_{c 4}+\left(A^{2}+B^{2}\right) \beta(\beta-1) E_{c}^{2}+2 A B \beta^{2} E_{c}^{2}=\left(A^{2}+B^{2}\right) \beta\left(E_{c 4}+(\beta-1) E_{c}^{2}\right)+2 A B \beta^{2} E_{c}^{2} \text {, } \\
& E\left[X^{2} \mid b_{l}=+1\right]=C^{2} \sum_{k=1}^{\beta} E\left[x_{k-\beta, l}^{2}\right] E\left[x_{(k-1)-\beta, l}^{2}\right]+C^{2} \sum_{k=1}^{\beta} E\left[x_{k-\beta, l} x_{(k-1)-\beta, l}\right] \times \sum_{m=1}^{\beta-1} E\left[x_{m-\beta, l} x_{(m-1)-\beta, l}\right]=C^{2} \beta E_{c}^{2} \text {, } \\
& E\left[Y^{2} \mid b_{l}=+1\right]=D^{2} \sum_{k=1}^{\beta} E\left[x_{k-\beta, l}^{2}\right] E\left[\eta_{k, l}^{2}\right]+D^{2} \sum_{k=1}^{\beta} E\left[x_{k-\beta, l}^{2}\right] E\left[\eta_{k-\beta, l}^{2}\right]+E^{2} \sum_{k=1}^{\beta} E\left[x_{(k-1)-\beta, l}^{2}\right] E\left[\eta_{k, l}^{2}\right]+ \\
& E^{2} \sum_{k=1}^{\beta} E\left[x_{(k-1)-\beta, l}^{2}\right] E\left[\eta_{k-\beta, l}^{2}\right]=\left(D^{2}+E^{2}\right) \beta N_{0} E_{c} \\
& E\left[Z^{2} \mid b_{l}=+1\right]=\lambda^{2} \sum_{k=1}^{\beta} E\left[\eta_{k, l}^{2}\right] E\left[\eta_{k-\beta, l}^{2}\right]=\lambda^{2} \beta \frac{N_{0}^{2}}{4} \text {. }
\end{aligned}
$$

to the increment of the power consumption as well as the cost of hardware implementation. However, with a robust development of the IC technology as nowadays, we can implement the receiver more easily by means of the high speed, low consumption and low cost ICs. The hardware implementation and performance measurement to validate the obtained results are also our future works.

\section{Appendix A}

STATISTICAL MEANS AND SECOND MOMENTS OF THE COMPONENTS $W, X, Y, Z$

The means of the components $W, X, Y, Z$ are given in Equations (43), (44), (45), and (46) at the top of this page, respectively. Also, the second moments of $W, X$, $Y, Z$ are given in Equations (47), (48), (49), and (50), respectively.

\section{Appendix B}

\section{StATISTICAL PROPERTIES OF CHEbyseV} POLYNOMIAL FUNCTION OF ORDER 2

The equation for the Chebysev polynomial function of order 2 is

$$
x_{k}=f\left(x_{k-1}\right)=2 x_{k-1}^{2}-1,
$$

and the invariant PDF of $x$, denoted by $\rho(x)$ is

$$
\rho(x)= \begin{cases}\frac{1}{\pi \sqrt{1-x^{2}}}, & |x|<1, \\ 0, & \text { otherwise. }\end{cases}
$$

The values of $E_{c}$ and $E_{c 4}$ are calculated as

$$
\begin{aligned}
E_{c} & =E\left[x_{k, l}^{\left.(i)^{2}\right]}\right. \\
& =\int_{-\infty}^{\infty} x^{2} \rho(x) d x=\int_{-1}^{1} x^{2} \frac{1}{\pi \sqrt{1-x^{2}}} d x=\frac{1}{2} \\
E_{c 4} & =E\left[x_{k, l}^{(i)^{4}}\right] \\
& =\int_{-\infty}^{\infty} x^{4} \rho(x) d x=\int_{-1}^{1} x^{4} \frac{1}{\pi \sqrt{1-x^{2}}} d x=\frac{3}{8} .
\end{aligned}
$$

\section{REFERENCES}

[1] F. C. Lau and C. K. Tse, Chaos-based digital communication systems: operating principles, analysis methods, and performance evaluation. Springer, 2003.

[2] P. Stavroulakis, Chaos applications in telecommunications. CRC press, 2005.

[3] X. Q. Nguyen, T. Q. Bui, T. D. Nguyen, T. M. Hoang et al., "Simulation and implementation of improved chaotic colpitts circuit for uwb communications," in Third International Conference on Communications and Electronics (ICCE 2010). Nha Trang, Vietnam: IEEE, 2010, pp. 307312.

[4] J. Yu and Y.-D. Yao, "Detection performance of chaotic spreading lpi waveforms," IEEE transactions on wireless communications, vol. 4, no. 2, pp. 390-396, 2005.

[5] N. Xuan Quyen, V. Van Yem, T. Manh Hoang, and K. Kyamakya, "M× n-ary chaotic pulse-width-position modulation: An effective combination method for improving bit rate," COMPEL-The international journal for 
computation and mathematics in electrical and electronic engineering, vol. 32, no. 3, pp. 776-793, 2013.

[6] N. X. Quyen, V. Van Yem, T. M. Hoang, S. El Assad, and K. Kyamakya, "Combination scheme of cpwpm and bpsk for digital communication," in Fourth International Conference on Communications and Electronics (ICCE 2012). IEEE, 2012, pp. 190-195.

[7] N. X. Quyen, V. Van Yem, and T. M. Hoang, "Improvement of bit rate using m-ary chaotic pulse position modulation," REV Journal on Electronics and Communications, vol. 1, no. 03, 2011.

[8] L. Gong and L. Shaoqian, "Chaotic spreading sequences with multiple access performance better than random sequences," IEEE Transactions on Circuits and Systems I: Fundamental Theory and Applications, vol. 47, no. 3, pp. 394-397, 2000.

[9] G. KOLUMBaN, K. GaBOR, J. Zoltan, and M. P. Kennedy, "Fm-dcsk: A robust modulation scheme for chaotic communications," IEICE Transactions on Fundamentals of Electronics, Communications and Computer Sciences, vol. 81, no. 9, pp. 1798-1802, 1998.

[10] S. M. Berber and A. K. Gandhi, "Inherent diversity combining techniques to mitigate frequency selective fading in chaos-based dsss systems," Physical Communication, vol. 19, pp. 30-37, 2016.

[11] W. M. Tam, F. C. Lau, C. Tse, and A. J. Lawrance, “Exact analytical bit error rates for multiple access chaos-based communication systems," IEEE Transactions on Circuits and Systems II: Express Briefs, vol. 51, no. 9, pp. 473-481, 2004.

[12] G. Kaddoum, D. Roviras, P. Chargé, and D. FournierPrunaret, "Accurate bit error rate calculation for asynchronous chaos-based ds-cdma over multipath channel," EURASIP Journal on Advances in Signal Processing, vol. 2009, p. 48, 2009.

[13] N. X. Quyen, V. V. Yem, and T. M. Hoang, "A chaosbased direct-sequence/spread-spectrum communication scheme," in Proc. Int. Symp. on Theoretical Electrical Engineering (ISTET'13), Pilsen-Czech Republic. University of West Bohemia, 2013, pp. III-1-III-2.

[14] N. X. Quyen, N. H. Long, V. Van Yem et al., "An ofdmbased chaotic dsss communication system with m-psk modulation," in Fifth International Conference on Communications and Electronics (ICCE 2014). IEEE, 2014, pp. 106-111.

[15] S. M. Berber, "Probability of error derivatives for binary and chaos-based cdma systems in wide-band channels," IEEE Transactions on Wireless Communications, vol. 13, no. 10, pp. 5596-5606, 2014.

[16] N. X. Quyen, V. Van Yem, and T. Q. Duong, "Design and analysis of a spread-spectrum communication system with chaos-based variation of both phase-coded carrier and spreading factor," IET Communications, vol. 9, no. 12, pp. 1466-1473, 2015.

[17] G. Kolumbán, B. Vizvári, W. Schwarz, and A. Abel, "Differential chaos shift keying: A robust coding for chaos communication," in Proc. NDES'96, 1996, pp. 8792.

[18] F. C.-M. Lau, M. Yip, C. Tse, and S. F. Hau, "A multiple access technique for differential chaos shift keying," in The IEEE International Symposium on Circuits and Systems (ISCAS 2001), vol. 3. IEEE, 2001, pp. 317-320.

[19] W. Xu, L. Wang, and G. Chen, "Performance of dcsk cooperative communication systems over multipath fading channels," IEEE Transactions on Circuits and Systems I: Regular Papers, vol. 58, no. 1, pp. 196-204, 2011.

[20] G. Setti, R. Rovatti, and G. Mazzini, "Synchronization mechanism and optimization of spreading sequences in chaos-based ds-cdma systems," IEICE Transactions on Fundamentals of Electronics, Communications and Computer Sciences, vol. 82, no. 9, pp. 1737-1746, 1999.

[21] B. Jovic, C. Unsworth, G. S. Sandhu, and S. M. Berber, "A robust sequence synchronization unit for multi-user ds-cdma chaos-based communication systems," Signal Processing, vol. 87, no. 7, pp. 1692-1708, 2007.

[22] G. Kaddoum, D. Roviras, P. Chargé, and D. FournierPrunaret, "Robust synchronization for asynchronous multi-user chaos-based ds-cdma," Signal Processing, vol. 89, no. 5, pp. 807-818, 2009.

[23] R. Vali, S. M. Berber, and S. K. Nguang, "Effect of rayleigh fading on non-coherent sequence synchronization for multi-user chaos based ds-cdma," Signal Processing, vol. 90, no. 6, pp. 1924-1939, 2010.

[24] S. Mandal and S. Banerjee, "Analysis and cmos implementation of a chaos-based communication system," IEEE Transactions on Circuits and Systems I: Regular Papers, vol. 51, no. 9, pp. 1708-1722, 2004.

[25] M. Delgado-Restituto, A. J. Acosta, and A. RodríguezVázquez, "A mixed-signal integrated circuit for fm-dcsk modulation," IEEE journal of solid-state circuits, vol. 40, no. 7, pp. 1460-1471, 2005.

[26] M. Sushchik, L. S. Tsimring, and A. R. Volkovskii, "Performance analysis of correlation-based communication schemes utilizing chaos," IEEE Transactions on Circuits and Systems I: Fundamental Theory and Applications, vol. 47, no. 12, pp. 1684-1691, 2000.

[27] G. Kaddoum, P. Chargé, D. Roviras, and D. FournierPrunaret, "Performance analysis of differential chaos shift keying over an awgn channel," in International Conference on Advances in Computational Tools for Engineering Applications (ACTEA'09). IEEE, 2009, pp. 255-258.

[28] Z. Zhou, J. Wang, and Y. Ye, "Exact ber analysis of differential chaos shift keying communication system in fading channels," Wireless personal communications, vol. 53, no. 2, pp. 299-310, 2010.

[29] Y. Xia, C. Tse, and F. C. Lau, "Performance of differential chaos-shift-keying digital communication systems over a multipath fading channel with delay spread," IEEE Transactions on Circuits and Systems II: Express Briefs, vol. 51 , no. 12 , pp. $680-684,2004$

[30] Y. Xia, C. K. Tse, F. C. Lau, and G. Kolumbán, “Performance of frequency-modulated differential-chaos-shiftkeying communication system over multipath fading channels with delay spread," International Journal of Bifurcation and Chaos, vol. 15, no. 12, pp. 4027-4033, 2005.

[31] Z. Zhou, T. Zhou, and J. Wang, "Performance of multiple-access dcsk communication over a multipath fading channel with delay spread," Circuits, Systems $\mathcal{E}$ Signal Processing, vol. 27, no. 4, pp. 507-518, 2008.

[32] T. S. Rappaport et al., Wireless communications: principles and practice. Prentice Hall PTR New Jersey, 1996, vol. 2.

[33] F. C. Lau, K. Y. Cheong, and C. Tse, "Permutationbased dcsk and multiple-access dcsk systems," IEEE Transactions on Circuits and Systems I: Fundamental Theory and Applications, vol. 50, no. 6, pp. 733-742, 2003.

[34] H. Yang and G.-P. Jiang, "Reference-modulated dcsk: a novel chaotic communication scheme," IEEE Transactions on Circuits and Systems II: Express Briefs, vol. 60, no. 4, pp. 232-236, 2013.

[35] G. Kaddoum, F.-D. Richardson, and F. Gagnon, "Design and analysis of a multi-carrier differential chaos shift keying communication system," IEEE Transactions on Communications, vol. 61, no. 8, pp. 3281-3291, 2013.

[36] Y. Fang, L. Wang, P. Chen, J. Xu, G. Chen, and W. Xu, "Design and analysis of a dcsk-arq/carq system over multipath fading channels," IEEE Transactions on Circuits and Systems I: Regular Papers, vol. 62, no. 6, pp. 1637-1647, 2015.

[37] G. Kaddoum, E. Soujeri, C. Arcila, and K. Eshteiwi, “Idcsk: an improved noncoherent communication system architecture," IEEE Transactions on Circuits and Systems II: Express Briefs, vol. 62, no. 9, pp. 901-905, 2015.

[38] L. Wang, X. Min, and G. Chen, "Performance of simo fmdcsk uwb system based on chaotic pulse cluster signals," IEEE Transactions on Circuits and Systems I: Regular Papers, vol. 58, no. 9, pp. 2259-2268, 2011. 
[39] Y. Fang, J. Xu, L. Wang, and G. Chen, "Performance of mimo relay dcsk-cd systems over nakagami fading channels," IEEE Transactions on Circuits and Systems I: Regular Papers, vol. 60, no. 3, pp. 757-767, 2013.

[40] S. Berber and N. Chen, "Physical layer design in wireless sensor networks for fading mitigation," Journal of Sensor and Actuator Networks, vol. 2, no. 3, pp. 614-630, 2013.

[41] IEEE 802 Working Group and others, "Ieee standard for local and metropolitan area networksÜpart 15.4: Lowrate wireless personal area networks (lr-wpans)," IEEE Std, vol. 802, pp. 4-2011, 2011.

[42] J. G. Proakis, Ed., Digital Communications, 4th ed. McGraw-Hill, New York, NY, USA, 2000.

[43] E. N. Gilbert, "Increased information rate by oversampling," IEEE transactions on information theory, vol. 39, no. 6, pp. 1973-1976, 1993.

[44] B. El-Khaldi, J. M. Rouvaen, A. Rivenq, and Y. El-Hillali, "Averaging and oversampling correlator receiver with input quantization," Digital Signal Processing, vol. 16, no. 2, pp. 120-136, 2006

[45] Y.-S. Lee and B.-S. Seo, "Ofdm receivers using oversampling with rational sampling ratios," IEEE Transactions on Consumer Electronics, vol. 55, no. 4, pp. 1765-1770, 2009.

[46] N. H. Long, N. X. Quyen, and V. Van Yem, "A direct sampling receiver for communication of dcsk systems over flat fading channels," in International Conference on Advanced Technologies for Communications (ATC 2015). IEEE, 2015, pp. 588-593.

[47] G. Kaddoum, P. Chargé, and D. Roviras, "A generalized methodology for bit-error-rate prediction in correlation- based communication schemes using chaos," IEEE Communications letters, vol. 13, no. 8, pp. 567-569, 2009.

[48] G. Kaddoum, F. Gagnon, P. Chargé, and D. Roviras, "A generalized ber prediction method for differential chaos shift keying system through different communication channels," Wireless Personal Communications, vol. 64, no. 2, pp. 425-437, 2012.

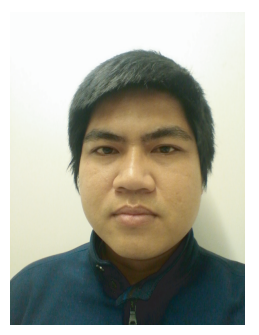

Nguyen Xuan Quyen received the Diploma of Engineer, Master and $\mathrm{PhD}$ degrees in Electronic Engineering and Telecommunications, from Hanoi University of Science and Technology, Vietnam in 2006, 2008 and 2013, respectively. From 2007-current, he has been serving as a senior lecturer at the School of Electronics and Telecommunications (SET), Hanoi University of Science and Technology (HUST), Vietnam. He has been a sandwich PhD student at the Institute for Smart System Technologies, Alpen-Adria Klagenfurt University, Austria during 2011-2012. From 2014-2015, he has worked as a postdoctoral researcher at the Department of Computer Architecture, Polytechnic University of Catalonia (UPC BarcelonaTech), Barcelona, Spain. He was also an academic visitor at the Department of Electronic and Computer Engineering, University of Limerick, Ireland in 2015 and School of Electronics, Electrical Engineering and Computer Science, Queen's University Belfast, United Kingdom in 2016. His main research interests are microwave engineering, chaos-based digital communications and physical layer security, and wireless communications. 